\title{
El descubrimiento de una sobreviviente: cepa vinífera Tamarugal
}

\author{
The discovery of a survivor: Tamarugal grapevine variety \\ Ingrid Poblete $^{1 *}$, Marcelo Lanino ${ }^{1}$, Gonzalo Rojas ${ }^{2}$
}

\section{RESUMEN}

En la región de Tarapacá se produjo vino desde el siglo XVI, y era una actividad importante en la localidad de Pica y Matilla, en la cual se obtuvieron aproximadamente 350.000 litros. Sin embargo, producto de varios factores, entre ellos el término del ciclo salitrero, la competencia vitivinícola desde la zona central, la aplicación de elevados impuestos y la expropiación de las aguas para el uso de la ciudad de Iquique, se inició el arranque de las plantas de vid. La actividad vitivinícola terminó entre 1937 y 1949 . No obstante, quedaron algunas plantas relictas en distintas localidades de la región, que sobrevivieron a las condiciones adversas del desierto más árido del mundo, como son altas temperaturas diurnas durante todo el año, baja humedad relativa, elevada radiación solar, ausencia casi absoluta de precipitaciones y suelo salino. Por ello, se efectuó un rescate de este material vegetal, estableciéndose un pequeño Jardín de Variedades en la Estación Experimental Canchones de la Universidad Arturo Prat. Se realizó un análisis molecular (ADN), a nivel nacional e internacional, para identificar a qué cepas correspondían. Finalmente quedó un genotipo sin identificar, después de haberlo contrastado con aproximadamente 7.000 genotipos. Se utilizó esta información como antecedente complementario para el registro de la cepa a nivel del Servicio Agrícola y Ganadero (SAG).

Palabras clave: historia vitivinícola, Vitis vinífera, desierto, Pampa del Tamarugal, perfil molecular.

\begin{abstract}
Since the XVI century, wine has been produced in the Tarapacá region, being an important activity in the town of Pica and Matilla, in which it was possible to obtain approximately 350,000 Liters. However, as a result of several factors, including the end of the saltpeter cycle, competition from the central zone, the application of high taxes, and the expropriation of water for the use of the city of Iquique, the vine plants began to be removed. The winemaking activity ended between 1937 and 1949. However, some relict plants remained in different locations in the region, which survived the driest desert's adverse conditions, such as high daytime temperatures throughout the year, low relative humidity, high solar radiation, and almost absolute absence rainfall, and saline soil. Therefore, a rescue of this plant material was carried out, establishing a small Variety Garden in the Canchones Experimental Station of the Arturo Prat University. At a national and international level, a molecular analysis (DNA) was carried out to identify which strains corresponded to them. A genotype could not be identified after it had been compared with approximately 7,000 genotypes. This information was used as a complementary antecedent for the registration of the variety at the Agricultural and Livestock Service (SAG) level.
\end{abstract}

Keywords: viticultural history, Vitis vinífera, desert, Pampa del Tamarugal, molecular profile.

\section{La historia del vino en la región de Tarapacá}

\section{Origen de la vitivinicultura sudamericana}

En la actualidad, existe un amplio consenso entre los historiadores sobre el origen de la vitivinicultura sudamericana. En su obra "Las viñas de Lima: inicios de la vitivinicultura sudamericana", el historiador peruano Guillermo Toro-Lira expone: "El primero que escribió sobre la introducción de la vid en el Perú fue el fraile Vicente de Valverde, primer obispo del Cusco y de Sudamérica, quien, a principios de 1539 señaló: Muy extremada tierra para viñas, a lo que todos nos parece, esperándose ahora plantas de vides, que se traerán desde Guatemala" (Toro-Lira, 2018).

Lorenzo Huertas sostiene que los primeros en cultivar la vid en el Perú fueron el propio Francisco Pizarro y Hernando de Montenegro, ambos en Lima. Desde este centro primigenio

\footnotetext{
1 Facultad de Recursos Naturales Renovables, Universidad Arturo Prat, Iquique, Chile.

2 Gonzalo Rojas Aguilera, Vinífera Chile, Santiago, Chile.

* Autor para correspondencia: ingrid.poblete@unap.cl
}

Fecha de Recepción: 1 de Junio, 2020.

Fecha de Aceptación: 30 de Septiembre, 2020. 
de la vitivinicultura sudamericana se habrían llevado las vides, durante la década de 1540, hacia el sur del Perú y Chile, en expediciones sucesivas, que fueron expandiendo el cultivo en las incipientes colonias. En este contexto, tal como ocurriría en el hemisferio norte de América, los primeros conquistadores extendieron el cultivo de la cepa Listán Negro, originaria de Castilla, que previamente había sido llevada a las Islas Canarias, Las Antillas y Mesoamérica, a estos dos últimos sitios, por Cristóbal Colón, primero, y Hernán Cortés, después. Denominada como Criolla en Perú, en Chile adquirió tempranamente el nombre de Cepa del País, para más tarde quedar establecida como Cepa País, la cepa fundacional del vino americano.

Según el trabajo de Guillermo Toro-Lira, quien ha recogido los aspectos más significativos de la historiografía vitivinícola peruana, no cabe duda de que el primer viticultor del Perú fue Hernando de Montenegro, que habría plantado las primeras vides -traídas desde la Capitanía General de Guatemala- en 1539, obteniendo la primera cosecha en 1541. Asimismo, el historiador José Busto, basándose en los documentos coloniales del Perú, sostiene: "La primera persona que plantó viñas en estas tierras y otros muchos árboles de Castilla, y que todas plantas cuanto hay hasta Chile, han tenido como origen la huerta y viña de Hernando de Montenegro" (Toro-Lira, 2008).

Para comprender el origen de la vitivinicultura sudamericana, es preciso tener en consideración la importancia fundamental que la cultura hispánica le asignaba al vino como producto cultural. Elemento esencial de la misa cristiana y la evangelización, el vino es considerado también como un alimento básico de la dieta mediterránea, extraordinariamente apetecido por los hispanos, tanto por su valor nutritivo como por sus características organolépticas. Ya sea mezclado con agua (para hidratarse de manera segura) o bien solo o con frutas, el consumo de vino está naturalizado en lo más fundamental de la alimentación de los colonos españoles, quienes lo utilizaban además para las expediciones militares de conquista, entregando raciones periódicas a las tropas.

Con una dimensión múltiple dentro de la cultura hispánica, la importancia del vino no sólo se circunscribe al ámbito religioso, gastronómico o militar, sino también a la dimensión más privada de la cultura, como las celebraciones íntimas de cada familia y amigos. Es por ello que, rápidamentexx en la América colonial, este producto fue adquiriendo una dimensión económica de gran relevancia, lo cual explica por qué, allí donde se asentaban los hispanos, inmediatamente comenzaba el cultivo de vides.

\section{La llegada del vino a Chile}

Todo indica que el origen de la vitivinicultura en Chile tiene como primer actor al conquistador Pedro de Valdivia. Si bien existe la referencia anterior a la expedición de Pedro de Valdivia, llevada a cabo por Diego de Almagro y su hueste en 1536, aún no hay mayores registros documentales de su paso por las tierras de Tarapacá, donde se indique una eventual contribución en torno al origen de la vitivinicultura. Esta es una temática que aún necesita ser estudiada con mayor profundidad (Nota de los autores).

En efecto, no solamente se le reconoce la primera referencia escrita y documentada sobre la existencia de vino en Chile, sino además, la orden de traer las primeras vides a la Capitanía General de Chile. Sin embargo, los historiadores chilenos sostienen, con bastante consenso, que el primer colono en dedicarse a la vitivinicultura en Chile habría sido Rodrigo de Araya, cuya primera vendimia dataría de 1551. Araya fue secundado prontamente por varios otros conquistadores, entre los que se cuentan Juan Jufré, Francisco de Aguirre y Rodrigo de Quiroga, quienes habrían expandido la vitivinicultura por las diversas comarcas de Chile en una época muy temprana, en torno a 1551 y 1600 (Lacoste, 2019).

Efectivamente, para fines del siglo XVI, ya se documenta la existencia de vides viníferas en lugares tan alejados como el valle de Copiapó, por el norte, hasta el valle del Bío-Bío, por el sur. El propio Valdivia se dedicaría a cultivar sus viñedos en su hacienda de Quilicura, un conocimiento que ha ido acumulándose con los trabajos de diversos cronistas y estudiosos coloniales, como Alonso González de Nájera, Gerónimo de Vivar, Diego de Rosales y Alonso de Ovalle. La mayor parte de estos estudios fueron sistematizados por el naturalista francés Claude Gay hacia mediados del siglo XIX, y han servido como base fundamental para la historiografía chilena en esta materia.

Con respecto al origen del vino chileno, en épocas más recientes destacan varios trabajos 
académicos (Del Pozo, 2014; Rojas, 2015; Lacoste 2019), los cuales han permitido actualizar y complementar el conocimiento en la materia. Sin embargo, todavía existen enormes desafíos que afrontar en este ámbito, lo que hace necesario un continuo ejercicio de investigación y análisis de las fuentes documentales de la era colonial.

\section{Los ciclos de la vitivinicultura en Tarapacá: el gran ciclo económico de la minería colonial}

A diferencia de lo que ocurre con el origen y expansión de la vitivinicultura en el denominado Valle Central, en la región de Tarapacá se experimentó otro acontecer histórico, prácticamente separado del resto del territorio chileno hasta finales del siglo XIX. Ligada administrativamente al Virreinato del Perú, primero, y a la República del Perú hasta 1883, la historia de la región estuvo íntimamente conectada con el gran ciclo económico expansivo de la minería colonial, durante prácticamente todo el período. Este vínculo posibilitó el desarrollo de la vitivinicultura en los oasis y quebradas de Tarapacá, en la medida en que los principales centros mineros cercanos incrementaban su demanda de alcohol. Este hecho obedece al primer ciclo en la historia vitivinícola tarapaqueña, marcado por el origen y desarrollo de la vitivinicultura colonial. El oasis de Pica y luego Matilla fueron los primeros lugares de asentamiento español, para desarrollar la agricultura que pedía la población en el yacimiento de plata de Huantajaya. Seguidamente, a fines del siglo XVI, la explotación de yacimientos de plata en Potosí produjo gran demanda de productos agrícolas, en especial, de vino.

Existe consenso entre los especialistas en destacar a las localidades de Pica y Matilla como los primeros lugares de asentamiento español, donde comenzó el desarrollo agrícola criollo y mestizo, tras la invasión hispánica. "Hacia fines del siglo XVI, se hacía urgente la demanda de vinos. Entonces el gobierno virreinal promueve la inmigración más al sur de Arequipa, con la real orden expedida en octubre de 1591 y dirigida al Virrey García Hurtado de Mendoza, indica la necesidad de "animar a la gente que reside en la tierra sin vecindad propia, tratos ni granjerías para que vayan a poblar Charcas y Atacama, pues en ciento ochenta leguas no hay pueblo de españoles y además de aquellas tierras es buena para el cultivo de viñas y las gentes las podría y viviría allí de buena gana" (Bermúdez, 1973).

Daponte (2006) señala: "De esta manera comienzan los primeros cultivos de viñas en la zona, como dan cuenta los escritos por el Arcediano Echeverría y Morales. Entre 1560 y 1620, paulatinamente la villa de Pica se consolida y estructura como pueblo; las construcciones aumentan y se intensifica la actividad social basada en la economía de haciendas y la producción vitivinícola. Esta bonanza económica atrajo a los "de pura estirpe española" a asentarse alrededor del entonces declarado por la iglesia, "pueblo de indios" de Pica, donde por una cédula real emitida a mediados del siglo XVIII, no podían habitar españoles ni sus esclavos. Haciendo crecer la hacienda de Matilla".

En otro estudio se da especial atención al Lagar de Matilla, hoy Monumento Histórico Nacional. "Ensanchando mediante sucesivas adquisiciones de tierras labrantías y puquios, las propiedades que su noble esposa poseyó en aquél partido, el dicho Gaspar de Loayza fundó una hacienda de viso, que así fue de pan llevar como de viña, a la que aplicó el nombre de Matilla en recuerdo de la Dehesa de la Umbría de España y la dotó de casa habitación lujosa y cómoda y de múltiples dependencias, en que vivieron vida cristiana y holgada su descendencia, relaciones dependientes y esclavos, poco a poco al subdividirse la dicha propiedad del fundador y sus herederos, se constituyó alrededor de la casa solariega y de la iglesia mandada a construir por Don Gaspar, un verdadero pueblo; el de San Antonio de Matilla de nuestros días" (Advis, 1994: 35, cit. a Cuneo Vidal, 1978: 285).

A inicios del siglo XVII se puede ver cómo aparecen en los documentos "Gaspar de Loayza y otros con sus familias en la localidad de Matilla, cultivando las primeras vides, frutales y crianza de animales. Las primeras familias españolas que plantaron viñas en Matilla y Pica para producir vino, obtuvieron sus títulos en Arica en 1618" (Calle, 2019).

"El descubrimiento y desarrollo del gran mineral de Plata de Potosí en el Alto Perú en las últimas décadas del siglo XVI trajo como consecuencia el decaimiento de Huantajaya y, por ende, la disminución productiva y un paulatino abandono de Camiña y Tarapacá, que dependían de la demanda argentífera de Huantajaya. Los 
pueblos de indios al sur del curato Tarapacá como Pica y sus anexos, que debido a sus ventajas naturales hídricas y agrícolas presentaban un mejor proyecto agropecuario, especialmente vitivinícola, para Potosí. Para cumplir mejor con la acción evangelizadora, la Iglesia reorganizó el campo de acción doctrinal dividiendo el Obispado del Cuzco en dos entre 1609 y 1614, quedando la zona sur administrada por el nuevo obispado de Arequipa, cuya administración doctrinal funcionó con los mismos curatos" (Daponte, 2006).

El crecimiento demográfico de Pica, en especial el de la población española y sus esclavos que en un principio se asentaron en los alrededores del pueblo de indios de Pica, dando origen a las haciendas de Matilla y Quisma -que durante el siglo XVII se transformaron en pueblos-, hizo necesario que en 1620 el obispo de Arequipa dividiera el curato de Tarapacá en dos. Así nació el nuevo Curato de Pica, dependiente del obispado de Arequipa y del arzobispado de Lima.

Desde el siglo XVIII en adelante van surgiendo los datos cuantitativos de la producción vitivinícola de la zona. "Autores como Billinghurst (1886) afirman que la cantidad de botijas producidas al año para el siglo XVIII en toda la zona de Pica fue de 15.000 botijas, lo que equivaldría a 350.000 litros de vino aproximadamente y que fue disminuyendo paulatinamente, hasta la primera mitad del siglo XX" (Daponte, 2006). De esta manera, se puede concluir que el denominado "ciclo argentario" (ciclo histórico asociado a la producción de la plata en América del Sur) jugó un papel clave en el desarrollo vitivinícola de Tarapacá, al igual que en los demás dominios hispánicos circundantes a Potosí.

\section{La incorporación a la República de Chile y el ciclo salitrero}

Tras la independencia de las repúblicas de Perú y Chile, la región de Tarapacá experimentó un escaso desarrollo material y cultural, manteniéndose casi inalterada su estructura social mestiza y principalmente dependiente de la minería, y en menor medida, de la agricultura de subsistencia. En este sentido, se señala: "El reducido número de habitantes en el Iquique colonial puede también entenderse por las continuas epidemias que asolaron Tarapacá en 1717, 1758 y 1804. Este último año la fiebre amarilla causó la muerte de catorce residentes en el puerto, obligando al resto de la población a marcharse. Un informe de 1806 reporta una población de apenas cuarenta personas; tres años después el número de habitantes había subido a cien, distribuidos ya entonces en los dos barrios más representativos de la ciudad: La Puntilla (extranjeros) y El Morro (indígenas y mestizos)" (Donoso, 2003).

Sin embargo, tras la incorporación forzosa del territorio tarapaqueño a Chile, con el consiguiente fenómeno de la "chilenización", las condiciones de los habitantes experimentaron grandes transformaciones. La principal de ellas, sin lugar a dudas, fue producto de la expansión de la economía salitrera.

Hacia fines del siglo XIX, el salitre representaba el principal producto minero del país, con un enorme peso en la economía chilena. En el centro de dicho fenómeno estuvo la floreciente ciudad de Iquique, antiguo villorrio de pescadores peruanos que rápidamente había pasado a convertirse en una dinámica ciudad, gracias a la producción y exportación de salitre. Sitios monumentales como las oficinas salitreras de Humberstone y Santa Laura, ambas reconocidas por la UNESCO como Patrimonio Cultural de la Humanidad, son el fiel testigo de aquella época, que tuvo sus últimos latidos hacia mediados del siglo XX.

La riqueza del salitre tuvo un efecto dinamizador sobre el conjunto de la economía chilena. Ciudades como Iquique y Antofagasta se convirtieron en grandes núcleos urbanos, que demandaban diversos alimentos, entre ellos, el vino. En este contexto, tiene una importancia significativa la llegada de algunos inmigrantes europeos a la región, entre los que destacan Heinrich Froehlich y Peter Müfeller.

Froehlich llegó a vivir a la ciudad de Iquique en 1923. En 1929 comenzaron los primeros intentos para desarrollar la agricultura en La Huayca, en sociedad con el inmigrante alemán Peter Muffeler. El trabajo entre ambos consistió en cultivar vides, frutas y verduras, además de criar aves y cerdos, para abastecer a la población de las oficinas salitreras. En 1936 Muffeler y Froehlich adquirieron la propiedad de Canchones, a la que denominaron Viña Los Puquios. "Froelich fue un investigador incansable, que pudo delimitar una chacra en Los Puquios, donde plantó las primeras vides que produjeron un vino generoso" (Calle, 2019).

Este inmigrante alemán logró desarrollar, no sin contratiempos, su proyecto vitivinícola 
en Canchones, elaborando una especie de vino fortificado denominado Tipo Oporto, el cual rápidamente adquirió fama en la región salitrera. En 1938, el gobierno del presidente Pedro Aguirre Cerda condecoró a Froehlich como caballero de la Orden al Mérito, por su contribución al desarrollo agrícola de la región de Tarapacá. En 1943, la recientemente creada Corporación de Fomento de la Producción de Chile se interesó por la experiencia agrícola de Froehlich en Los Puquios, y lo contrató para replicar su experiencia en otros poblados cercanos. Tenían como base la Estación Experimental Canchones, hoy propiedad de la Universidad Arturo Prat.

"Después de la década de 1930-1940 hasta nuestros días, la actividad vitivinícola decayó, cambiando drásticamente hacia la producción frutícola (naranjas, mangos, guayabas y el limón, entre otras), abasteciendo en un principio a las últimas oficinas salitreras y últimamente a las ciudades de Iquique, Calama y Antofagasta" (Daponte, 2006).

La última vendimia registrada en esta zona data de 1937, en el Lagar de Matilla y en 1949 en Canchones, Pampa del Tamarugal, donde se ubicaba la Viña Los Puquios. Luego, tras el cierre de las oficinas salitreras de la región -Humberstone en 1960 y Santa Laura en 1961 - y la muerte de Heinrich Froehlich en 1966, los últimos vestigios de esta época se llevaron consigo la tradición vitivinícola de Tarapacá, la que paulatinamente fue quedando en el olvido, relegada a algunos pequeños cultivos domésticos en los oasis y quebradas de la región, y en Canchones, donde hasta el día de hoy se guarda la memoria de Heinrich Froehlich y Peter Müfeller como pioneros de la vitivinicultura republicana del Norte Grande de Chile.

Según diversos autores tarapaqueños (Núñez, 1985; Daponte, 2006; Calle, 2019), los principales factores que explican el declive de la producción vitivinícola en Tarapacá son el fin del ciclo salitrero, con el consiguiente y relativo despoblamiento del interior de la región; la competencia asimétrica provocada por los vinos de la zona central del país, que ya para mediados del siglo XX se comercializaban a bajos precios en Iquique y otras ciudades del interior de la región; la aplicación de altos impuestos y la expropiación de las aguas comunales de Pica y Matilla para el uso de la ciudad de Iquique, con la consiguiente y mayoritaria eliminación de los cultivos viníferos tradicionales de la localidad, sustituyéndolos por otros más lucrativos, como los cítricos.

\section{Antecedentes edafoclimáticos de la Pampa del Tamarugal, región de Tarapacá}

\section{Características climáticas}

Di Castri y Hajek (1976), Santibáñez (1982) y Novoa et al. (1989) reportan que el clima predominante en la zona es de tipo desértico normal, con la denominación BW según Köppen. De acuerdo con registros para la década de 1940 (Tabla 1), se presentan temperaturas máximas en el mes más cálido de $32{ }^{\circ} \mathrm{C}$, mientras que las temperaturas mínimas del mes más frío son inferiores a $0{ }^{\circ} \mathrm{C}$. Santibáñez et al. (1982) señalan que la Pampa no es homogénea desde el punto de vista térmico y presenta áreas más frías que otras.

Desde el paralelo $18^{\circ}$ al paralelo $32^{\circ}$ LS se extiende la zona en la que se encuentra la Pampa del Tamarugal (Novoa et al., 1989). La influencia principal la constituye el denominado Anticiclón del Pacífico, que genera un área de escasa precipitación en Chile, por lo que estas zonas requieren de riego. También se debe considerar que existen variaciones

Tabla 1. Antecedentes climáticos históricos de la Pampa del Tamarugal, Canchones, período 1942-1948.

\begin{tabular}{|c|c|c|c|c|c|c|c|c|c|c|c|c|c|}
\hline & ENE & FEB & MAR & ABR & MAY & JUN & JUL & AGO & SEP & OCT & NOV & DIC & Pro \\
\hline $\mathrm{T}^{\circ}$ Máx & 31,9 & 32,3 & 32,1 & 31,2 & 29,8 & 28,5 & 29,0 & 30,1 & 31,3 & 31,3 & 31,8 & 31,9 & 30,9 \\
\hline T$^{o}$ Mín & 11,4 & 11,3 & 8,0 & 5,4 & 2,2 & 0,0 & $-0,2$ & $-0,6$ & 2,3 & 3,2 & 4,5 & 7,0 & 4,5 \\
\hline $\mathrm{T}^{\mathrm{o}}$ Prom & 21,0 & 21,0 & 19,2 & 17,0 & 14,6 & 13,5 & 12,8 & 13,4 & 15,7 & 16,4 & 17,6 & 19,0 & 16,8 \\
\hline HR (\%) & 52,0 & 53,0 & 54,0 & 54,0 & 53,0 & 52,0 & 50,0 & 47,0 & 45,0 & 46,0 & 47,0 & 49,0 & 50,2 \\
\hline $\mathrm{Pp}(\mathrm{mm})$ & 0,2 & 0,3 & 0,0 & 0,0 & 0,0 & 0,0 & 0,1 & 0,0 & 0,0 & 0,0 & 0,0 & 0,0 & $0,6(*)$ \\
\hline Hr sol d ${ }^{-1}$ & 11,3 & 10,4 & 10,4 & 10,0 & 8,3 & 8,1 & 10,1 & 10,1 & 11,1 & 10,8 & 11,6 & 11,5 & 10,3 \\
\hline
\end{tabular}

Fuente:Santibáñez et al., 1982.

(*): Corresponde al total acumulado anual. 
de este a oeste, debidas al relieve y la influencia del mar. Las precipitaciones reportadas son inferiores a $5 \mathrm{~mm}$ anuales, erráticas en el tiempo y sin una distribución definida (Santibáñez, 1982). Registros publicados para el sector de Canchones señalan un valor de precipitaciones promedio de $0,6 \mathrm{~mm}$ año ${ }^{-1}$ (Di Castri y Hajek, 1976; Santibáñez et al., 1982).

En verano el centro de presiones se detecta en el paralelo $30^{\circ} \mathrm{LS}$, a la altura de la ciudad de La Serena, mientras que en invierno se desplaza al norte, entre Taltal y Tocopilla entre los paralelos $20^{\circ}$ y $25^{\circ} \mathrm{LS}$. En invierno afecta hasta la latitud $38^{\circ}$ sur y en verano a los $42^{\circ} \mathrm{LS}$.

\section{Temperaturas}

Datos actualizados y con mayores antecedentes de los registros meteorológicos, correspondientes a la Estación Experimental Canchones ( $20^{\circ} 26^{\prime}$ LS, $69^{\circ} 32^{\prime}$ LO) de la Universidad Arturo Prat, para el período 1995 a 2020, se observan en la Figura 1.

El promedio anual de las temperaturas registradas para este período es de $18,6^{\circ} \mathrm{C}$, lo que representa una diferencia de $1,8^{\circ} \mathrm{C}$ con respecto a lo reportado anteriormente (Tabla 1). El promedio anual de temperaturas máximas es de $31,7^{\circ} \mathrm{C}$ y el promedio de temperaturas mínimas anuales de $5,6^{\circ} \mathrm{C}$. Esto muestra una condición climática que no permite un análisis detallado, por lo cual se han incluido las temperaturas máximas y mínimas absolutas por mes, como eventos importantes para la agricultura. En este caso se puede determinar que las mayores temperaturas registradas ocurren en los meses de octubre $\left(42^{\circ} \mathrm{C}\right)$, noviembre, enero y febrero $\left(40^{\circ} \mathrm{C}\right)$. También es importante destacar que la temperatura mínima absoluta más intensa se presenta en julio $\left(-8,6^{\circ} \mathrm{C}\right)$. Sin embargo, aquellas que tienen riesgo de heladas comienzan en abril, con una menor incidencia de $-0,4^{\circ} \mathrm{C}$. En mayo se presentan heladas frecuentes, con una intensidad de hasta $-3,2{ }^{\circ} \mathrm{C}$, y descienden drásticamente en los meses de junio, julio y agosto, cuando se concentran las heladas más intensas de $-6,7^{\circ} \mathrm{C}$, $-8,6^{\circ} \mathrm{C}$ y $-4,9^{\circ} \mathrm{C}$, respectivamente. En septiembre aún se registran heladas de menor intensidad y en octubre disminuyen, pero en noviembre puede ocurrir una helada tardía de $-0,8{ }^{\circ} \mathrm{C}$ (Figura 1). Estas heladas no solo son severas en intensidad, sino también por la duración en horas día.

\section{Precipitaciones}

Con respecto a los registros de precipitaciones, en la Tabla 2 se observa el promedio histórico por mes y total acumulado.

Según estos antecedentes, se puede señalar que el promedio de precipitaciones ha aumentado desde $0,6 \mathrm{~mm}$ año-1 (Tabla 1) a 1,1 mm año-1 (Tabla 2). Esto representa un incremento, sin embargo, debido a la baja cantidad de precipitaciones, no es una buena medida de evaluación. En la Figura 2 se muestran las precipitaciones anuales que se han registrado para el período 1999-2020. Es así como existen años de ocurrencia de precipitaciones y otros con ausencia absoluta de ellas. Esto da la condición de desierto absoluto, atribuible a la baja

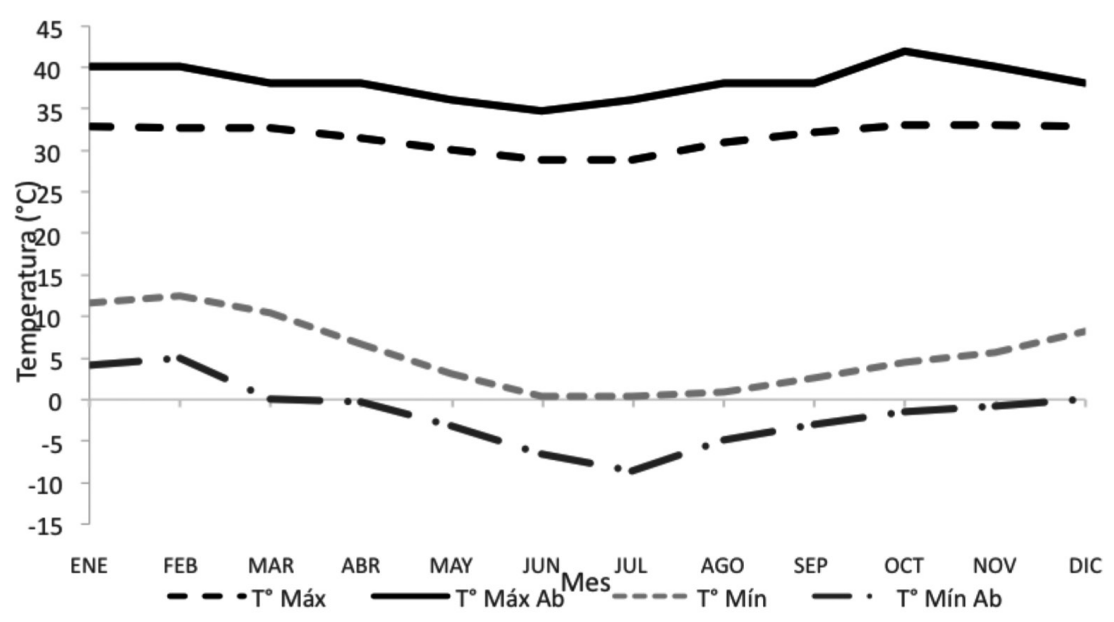

Figura 1. Temperaturas máximas y mínimas promedio y absolutas, Pampa del Tamarugal, período 1995-2020. Estación meteorológica, Estación Experimental Canchones. Universidad Arturo Prat. 
Tabla 2. Precipitaciones promedio por mes, período 1999-2020.

Estación meteorológica, Estación Experimental Canchones. Universidad Arturo Prat.

\begin{tabular}{|c|c|c|c|c|c|c|c|c|c|c|c|c|c|}
\hline & \multicolumn{13}{|c|}{ Mes } \\
\hline & ENE & FEB & MAR & $\mathrm{ABR}$ & MAY & JUN & JUL & AGO & SEP & OCT & NOV & DIC & Total \\
\hline Precipitaciones promedio $(\mathrm{mm})$ & 0,3 & 0,4 & 0,0 & 0,1 & 0,0 & 0,0 & 0,3 & 0,0 & 0,0 & 0,0 & 0,0 & 0,0 & 1,1 \\
\hline
\end{tabular}

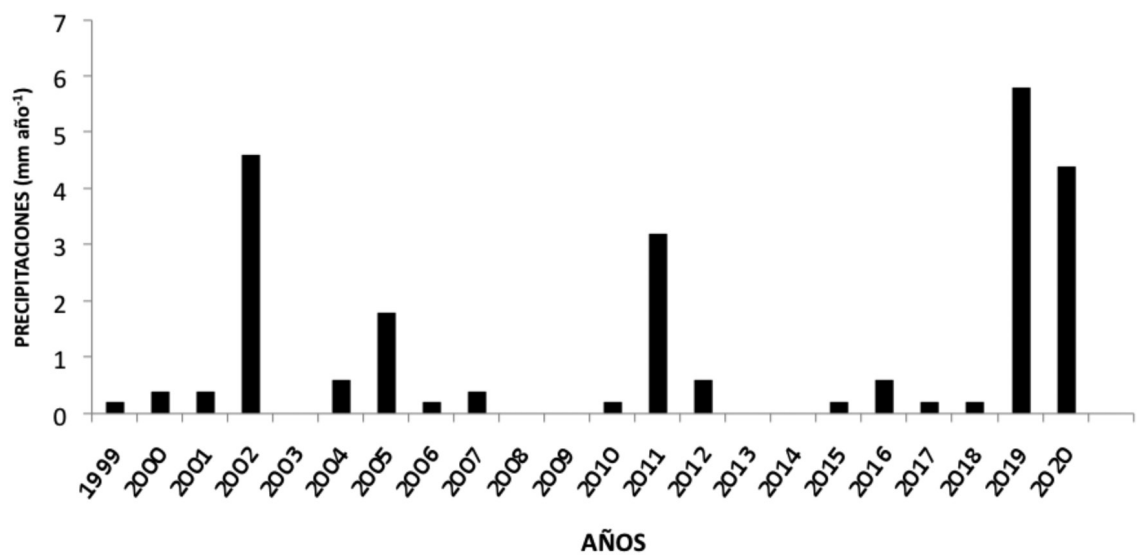

Figura 2. Precipitaciones anuales, período 1999-2020. Estación meteorológica, Estación Experimental Canchones, Universidad Arturo Prat.

cantidad total de precipitaciones y a lo eventual de estas. No obstante, en términos cuantitativos, existe un incremento histórico en el promedio anual de precipitaciones.

\section{Humedad relativa del aire}

La humedad relativa del aire (HR) representa un efecto importante para las plantas en las condiciones de la Pampa del Tamarugal, debido a la demanda evapotranspirativa. Los registros para el período 1999-2020 se indican en la Figura 3.

El promedio anual de la humedad relativa es de $63,2 \%$, con un máximo promedio de $85,8 \%$ y un mínimo promedio de $14,2 \%$. El valor máximo absoluto es de $97 \%$, que se registra en los meses de junio y julio, y el mínimo absoluto de $1 \%$ entre junio y septiembre. Sin embargo, durante todo el año se pueden encontrar valores diurnos inferiores a $7 \%$ (Figura 3).

\section{Radiación solar}

La radiación solar promedio anual reportada en este sector de la Estación Experimental Canchones varía entre 500 y $550 \mathrm{cal} \mathrm{cm}^{-2}$ día ${ }^{-1}$ (Santibáñez et al., 1982) o 20,93 a 23,03 $\mathrm{MJ} \mathrm{m}^{-2} \mathrm{~d}^{-1}$.

Para el período 1992-2020, la radiación solar promedio anual es de $20,8 \mathrm{MJ} \mathrm{m}^{2}$ día $^{-1}$, y los mayores valores se registran en noviembre $(26,5$ MJ $\mathrm{m}^{2}$ día $\left.^{-1}\right)$ y diciembre $\left(26,0 \mathrm{MJ} \mathrm{m}^{2}\right.$ día $\left.^{-1}\right)$, mientras que los menores en junio (14,5 $\mathrm{MJ} \mathrm{m}^{2}$ día ${ }^{-1}$ ) y julio (14,9 $\left.\mathrm{MJ} \mathrm{m}^{2} \mathrm{~d}^{-1}\right)$ (Tabla 3).

Estas condiciones ambientales representan una condición estresante para las plantas, sometidas a altas temperaturas, baja humedad ambiental, alta radiación y prácticamente nula precipitación.

\section{Caracterización de suelo de la Pampa del Tamarugal}

Con relación a las características del suelo, Santibáñez (1982) señala que el origen principal corresponde a mantos de rocas volcánicas terciarias, junto con depósitos sedimentarios terciarios y cuaternarios aluviales y lacustres, además de aluviones cuaternarios.

El ecosistema de la Pampa del Tamarugal se encuentra asociado a salares que presentan condiciones extremas en cuanto a suelos. Existen 


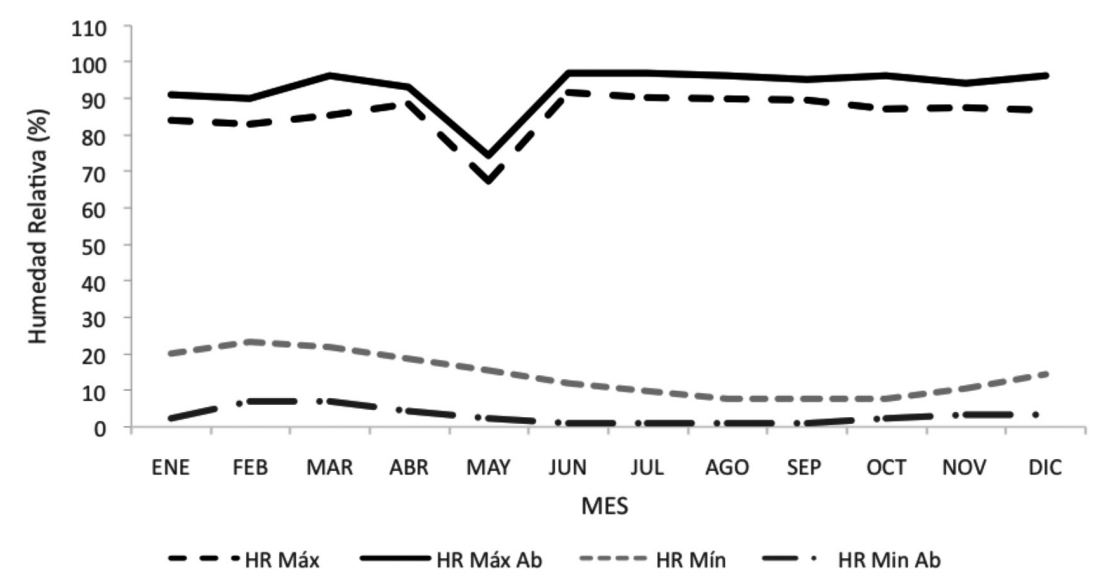

Figura 3. Humedad relativa (\%), mínima y máxima promedio y absoluta, período 1999-2020. Estación meteorológica, Estación Experimental Canchones, Universidad Arturo Prat.

Tabla 3. Radiación solar promedio (MJ m² día-1), período 1999-2020. Estación meteorológica, Estación Experimental Canchones, Universidad Arturo Prat.

\begin{tabular}{lllllllllllllll}
\hline & \multicolumn{11}{c}{ Mes } \\
\cline { 2 - 11 } & ENE & FEB & MAR & ABR & MAY & JUN & JUL & AGO & SEP & OCT & NOV & DIC & Prom. \\
\hline Radiación Solar $\left(\mathrm{MJ} \mathrm{m}^{-2} \mathrm{~d}^{-1}\right)$ & 24,8 & 23,6 & 21,7 & 18,7 & 15,8 & 14,5 & 14,9 & 16,4 & 21,4 & 24,8 & 26,5 & 26,0 & 20,8 \\
\hline
\end{tabular}

costras superficiales selladas, extremadamente duras y que, en algunos casos, pueden llegar a tener hasta 1,2 metros de espesor. Principalmente están constituidas por sulfatos, cloruros y carbonatos. Los $\mathrm{pH}$ del suelo son elevados, con valores que fluctúan entre 8 y 9 . Estos suelos tienen una topografía plana a suavemente ondulada con microrrelieve fuerte (Santibáñez et al., 1982; Acevedo et al., 2007).

Los suelos del sector Canchones son estratificados, con texturas que se alternan, y en ellos se encuentra desde arena hasta arcilla poco densa, con bajo contenido de materia orgánica $(0,01-0,57 \%)$. Son de colores claros y poseen una densidad aparente de 1,10 a $1,5 \mathrm{gr} \mathrm{cm}^{-3}$ (Acevedo et al., 2007). Presentan elevada conductividad eléctrica (CE) en el extracto de saturación, variando de 54 a $500 \mathrm{dSm}^{-1}$ en superficie. Y en las estratas más superficiales $(0 \mathrm{a} 20 \mathrm{~cm})$ poseen valores cercanos a $9 \mathrm{dS} \mathrm{m}^{-1}$, mientras que a mayor profundidad (20 a $40 \mathrm{~cm}$ ) se pueden encontrar valores que varían de 0,7 a $4,0 \mathrm{dS} \mathrm{m}^{-1}$. Esto considerando sectores que se hallan bajo riego, lo que muestra una alta capacidad de desplazamiento de las sales producto del agua (Lanino, 2012).
Con respecto al boro predominante, parece formar parte de la fracción móvil de las sales en el suelo, debido a que se moviliza mediante lixiviación con el agua de riego. Es elevado en la superficie $(0$ a $20 \mathrm{~cm})$ con valores cercanos a $8,5 \mathrm{meq} \mathrm{L}^{-1}$, previo al riego de lavado, y se reduce en forma importante, después del riego, a mayor profundidad (20 a $60 \mathrm{~cm}$ ), alcanzando valores de 0,81 meq L-1 (Lanino, 2012).

\section{Plantas antiguas de vid en la región de Tarapacá}

\section{Rescate de plantas antiguas}

La actividad vitivinícola de la región de Tarapacá, la cual se prolongó hasta el año 1937 en Matilla y 1949 en la Pampa del Tamarugal, como se mencionó anteriormente, desapareció por diversos factores que condujeron a la eliminación de las plantas de vid. Sin embargo, quedaron plantas relictas en distintas localidades de la región (Poblete et al., 2011). Estas plantas sobrevivieron a décadas de abandono, soportando condiciones estresantes, como suelos salinos, alta radiación y 
elevadas temperaturas diurnas durante el período de invierno (Figura 1, Tabla 3). En el caso de un frutal de clima templado, se afecta la brotación natural en primavera y por lo tanto la producción, además de la longevidad de la planta. Estas plantas hacen presente la historia vitivinícola de la región de Tarapacá, y también llevan consigo la información que las hizo resistir en las condiciones adversas del desierto más árido del mundo.

En el año 2003 la Universidad Arturo Prat inicia la colecta de estacas de plantas antiguas de vid (Vitis vinifera $\mathrm{L}$.), que se encontraban en lo que en ese entonces correspondía a la I Región. Las labores incluyeron un viñedo antiguo de la universidad ubicado en la Estación Experimental Canchones y que en la actualidad, de acuerdo a los últimos antecedentes, debe tener alrededor de 75 años. La colecta de estacas consideró plantas que visualmente se viesen añosas, tomando en cuenta el diámetro del tronco o que fuese indicado en forma oral por algún lugareño, dado que no existían antecedentes escritos. Estas plantas generalmente no presentaban manejo, y se encontraron algunas arbustivas, adosadas a un árbol, con madera envejecida. Ocasionalmente mostraban algún vestigio de pampanito; otras subsistían posiblemente por subirrigación, ya que no había sistema de riego por las inmediaciones, y otras se hallaban con algo de manejo, presentando un sistema en cabeza.

El nombre de estas variedades se perdió en el tiempo y solo se obtuvo, en algunos casos, el color de la baya. Estas estacas se enraizaron en la Estación Experimental Canchones, ubicada a 90 $\mathrm{km}$ al SE de Iquique y a $960 \mathrm{msnm}$. En el año 2004 se estableció un Jardín de Variedades de $2.000 \mathrm{~m}^{2}$, empleando un sistema de espaldera simple, con un marco de plantación de 2 × 1,5 m, con riego por goteo y con una orientación norte-sur.

Una vez establecidas, se comenzó la evaluación agronómica, para determinar su aptitud agrícola.
Se consideraron variables productivas y de calidad del racimo.

\section{Identificación molecular}

Al mismo tiempo, se inició un proceso de identificación molecular. Se empleó la técnica de marcadores de microsatélites, que permite identificar variedades, mediante el análisis de segmentos conocidos de ADN, altamente discriminantes para las variedades de vid. Estos marcadores flanquean segmentos cortos de ADN, de dos a seis nucleótidos, que se repiten con una alta frecuencia en el genoma y que son característicos de la variedad.

La evaluación, a nivel nacional, formó parte de la tesis doctoral de la suscrita. Esta primera etapa se llevó a cabo en el laboratorio de Biotecnología del Instituto de Investigaciones Agropecuarias (INIA), La Platina, a cargo del Dr. Patricio Hinrichsen, quien a su vez fue profesor colaborador de esta tesis doctoral.

En este primer análisis solo se identificó la cepa País, un genotipo bastante cosmopolita, que posee distintas sinonimias en el mundo. Se ha seguido cultivando en la zona centro-sur del país y también en el valle de Codpa (región de Arica y Parinacota), y está retomando importancia como cepa patrimonial.

Posteriormente, en el año 2010, continuando con el proceso de identificación molecular, en conjunto con el Dr. Patricio Hinrichsen, las muestras de ADN son enviadas al Instituto Madrileño de Investigación y Desarrollo Rural, Agrario y Alimentario (IMIDRA), España, en colaboración con la Dra. María Teresa de Andrés. En este instituto se utilizan 20 marcadores de microsatélites (Thomas and Scott, 1993; Bowers et al., 1996; Bowers et al., 1999; Laucou et al., 2011) (Tabla 4), los cuales se han empleado en otros estudios de identificación de variedades (Emanuelli et al., 2013; Ríaz et al., 2018). Se identifican dos genotipos: uno tinto, denominado Gros Colman,

Tabla 4. Perfil molecular de la cepa Tamarugal, originaria de la región de Tarapacá (IMIDRA; INRA).

\begin{tabular}{cccccccccc}
\hline CEPA & \multicolumn{1}{c}{ SSR } \\
\hline \multirow{5}{*}{ Tamarugal } & VVIB01 & VMC1B11 & VVIH54 & VVMD7 & VVMD24 & VVMD25 & VVIN73 & VVIP31 & VVIP60 \\
& $290-294$ & $167-189$ & $167-169$ & $237-247$ & $208-212$ & $237-247$ & $263-263$ & $176-192$ & $318-322$ \\
\cline { 2 - 9 } & VVIQ52 & VVS2 & VVMD5 & VVIN16 & VVMD32 & VVIV37 & VVMD28 & VMC4F3-1 & VVMD21 \\
& $82-88$ & $133-148$ & $225-229$ & 151-151 & $254-262$ & $163-175$ & $260-270$ & $203-206$ & $243-265$ \\
\cline { 2 - 9 } & VVMD27 & VVIV67 & & & & & & & \\
& $189-194$ & $352-375$ & & & & & & & \\
\hline
\end{tabular}


de Georgia, de la antigua Unión Soviética, cepa que tiene la particularidad de provenir de la zona de origen de la especie Vitis vinifera, en la región transcaucásica, entre el mar Caspio y el mar Negro; y uno rosado, llamado Ahmeur bou Ahmeur, originario de Argelia. Además se confirma la identificación de la cepa País y quedan dos genotipos blancos sin identificar (Poblete et al., 2011). Para el genotipo de bayas blancas y pequeñas se identifican sus padres, que corresponden a las cepas País y Moscatel de Alejandría. En cuanto al genotipo blanco de bayas grandes, se cree que puede ser descendiente de Moscatel de Alejandría o un hijo de este.

Posteriormente, en el año 2012, se toma contacto con el Instituto para la Investigación Agronómica (INRA), de Montpellier, Francia, con el Dr. Thierry Lacombe, quien analiza los pares de bases de todos los genotipos, identificados y sin identificar, previamente examinados por el IMIDRA.

En el INRA se logra identificar el genotipo blanco de bayas pequeñas, de nombre Torrontés Riojano, de origen argentino, el cual fue confirmado posteriormente también por el IMIDRA. El genotipo blanco de bayas grandes permaneció sin identificar tras ser contrastado con aproximadamente 7.000 genotipos, considerando las tres bases de datos.

Con estos antecedentes de Chile, España y Francia, complementados con informes de los tres países, en el año 2015 se presentan estos documentos al Servicio Agrícola y Ganadero (SAG) de Santiago, para proceder al registro de la cepa en Chile. Es importante señalar que esta información molecular constituye información adicional no definitiva. Este proceso de registro está enmarcado en los parámetros de la Unión Internacional para la Protección de las Obtenciones Vegetales (UPOV, 2008), organización intergubernamental con sede en Ginebra (Suiza), creada en París en 1961, que integran 76 países (UPOV, 2020), y corresponde al protocolo oficial para lograr la protección de una variedad.

Mediante este protocolo, que consiste en determinar tres rasgos en las plantas, como son distinción, homogeneidad y estabilidad (DHE), se obtiene el registro definitivo de la cepa en el año 2016 (Poblete, et al., 2017).

De esta manera se descubre la primera cepa vinífera chilena registrada, bautizada con el nombre de Tamarugal, considerando su cultivo en la Pampa del mismo nombre. Esta cepa, originaria de la región de Tarapacá.

\section{Conclusiones}

En la actualidad existe un amplio consenso sobre el origen de la vitivinicultura sudamericana, considerando a Lima como el núcleo central. Según Guillermo Toro-Lira, el primer viticultor del Perú fue Hernando de Montenegro, quien habría plantado las primeras vides en Lima, traídas desde la Capitanía General de Guatemala en 1539. La primera cosecha del Perú sería de 1541.

Con respecto a Chile, se destaca la figura del conquistador Pedro de Valdivia como el primer impulsor de la vitivinicultura en el país. Asimismo, el primer colono en dedicarse a la vitivinicultura en Chile habría sido Rodrigo de Araya, cuya primera vendimia dataría de 1551. Araya fue secundado prontamente por varios otros conquistadores, entre los que se cuentan Juan Jufré, Francisco de Aguirre y Rodrigo de Quiroga, quienes habrían expandido la vitivinicultura por las diversas comarcas de Chile en una época muy temprana, en torno a 1551 y 1600.

Por otra parte, en este artículo se exponen y analizan los ciclos de la vitivinicultura en Tarapacá, partiendo por el gran ciclo económico de la minería colonial, caracterizado por la llegada de los primeros cepajes desde el Perú y el asentamiento de los primeros colonos viñateros. Estos practicaron la vitivinicultura de manera sistemática durante el período colonial, como una forma de abastecer su consumo doméstico y de comerciar con los grandes centros mineros circundantes. Por consiguiente, se concluye que las primeras plantaciones de vides en Tarapacá tuvieron lugar en Pica y Matilla, en el siglo XVI.

La vitivinicultura de Tarapacá fue una industria importante. Sin embargo, diversos factores como el fin del ciclo salitrero, la competencia de asimétrica provocada por los vinos de la zona central del país, la aplicación de altos impuestos y la expropiación de las aguas comunales de Pica y Matilla para el uso de la ciudad de Iquique, llevaron al término de la actividad. La última vendimia para este período data de 1937 en la localidad de Matilla y 1949 en la Pampa del Tamarugal.

El desierto de Atacama, en la Pampa del Tamarugal, se caracteriza por presentar una alta oscilación térmica durante todo el año. La humedad relativa del aire se encuentra en valores muy reducidos, lo que representa una fuerte demanda de la atmósfera para las plantas. En igual sentido, la 
cantidad de energía solar incidente es alta durante gran parte del año, generando un ambiente de estrés. Las precipitaciones son muy reducidas y variables en el tiempo, lo que explica el concepto utilizado al referirse a la condición de desierto absoluto. Los suelos poseen costra salina, son estratificados, con presencia de alta concentración de sales y boro, lo cual dificulta el cultivo de especies agrícolas.

En la región se encontraron plantas antiguas de vid, en el año 2003, que lograron sobrevivir a estas condiciones de clima y suelo del desierto más árido del mundo. Por ello se efectuó un rescate de material vegetal, enraizándolo y estableciendo un Jardín de Variedades en la Estación Experimental Canchones de la Universidad Arturo Prat.

En este jardín, las plantas analizadas a nivel molecular tanto nacional como internacional, se encontró un genotipo que no contrastó con ningún patrón de ADN disponible. Esta información se utilizó como antecedente complementario para el registro de la cepa Tamarugal, la primera cepa vinífera chilena.

\section{Literatura Citada}

Acevedo, E.; Ortiz, M.; Franck, N.; Sanguinet, P.

2007. Relaciones Hídricas de Prosopis tamarugo Phil. Uso de Isótopos Estables. Serie Ciencias Agronómicas No 14/200. Santiago, Chile. 82 p.

Advis, $\mathrm{P}$.

1994. La doctrina de Tarapacá en el siglo XVI, perfil administrativo y eclesiástico. Tarapacá una aventura en el tiempo. Selecciones de Revista Camanchaca, Ediciones Camanchaca. Iquique, Chile. 86 p.

Bermúdez, O.

1973. Pica en el siglo XVIII. Estructura económica y social. Revista Chilena de Historia y Geografía 141: 7-56.

Billinghurst, G.

1886. Estudio sobre la geografía de Tarapacá. Páginas de un Libro. Trabajo escrito para El Ateneo de Iquique. Santiago, Imprenta de El Progreso. 113 p.

Bowers, J.E.; Dangl, G.S.; Vignani, R.; Meredith, C.P. 1996. Isolation and characterization of new polymorphic simple sequence repeat loci in grape (Vitis vinifera L.). Genome, 39: 628-633.

Bowers, J.E.; Dangl, G.S.; Meredith, C.P.

1999. Development and characterization of additional microsatellite DNA markers for grape. Am. J. Enol. Vitic., 50 (3): 243-246.

Calle, $\mathrm{M}$

2019. Historia de la vitivinicultura en la región de Tarapacá, siglos XVI-XX. En: UNAP. Enoturismo del Desierto. 2019. Iquique, Chile. pp. 18-31.

Daponte, J.

2006. El aporte de los negros a la identidad musical de Pica, Matilla y Tarapacá. Editorial Universidad de Chile. 89 p.

Del Pozo, J.

2014. Historia del vino chileno. Editorial Universitaria. Santiago, Chile. 376 p.

Di Castri, F.; Hajek, E.

1976. Bioclimatología de Chile. Vicerrectoría Académica de Universidad Católica de Chile. Santiago, Chile. 163 p.

Donoso, C.

2003. Fuentes raras y valiosas para la historia de Iquique. Revista de Ciencias Sociales, 13: 13-24.

Emanuelli, F.; Lorenzi, S.; Grzeskowiak, L.; Catalano, V.; Stefanini, M.; Troggio, M.; Myles, S.; Martínez-Zapater, J.; Zyprian., E.; Moreira, F.; Grando, S.
2013. Genetic diversity and population structure assessed by SSR and SNP markers in a large germoplasm collection of grape. BMC Plant Biology, 13: 39-55.

Lacoste, P.

2019. La vid y el vino en el Cono Sur de América: Argentina y Chile 1545-2019. Editorial RIL. Santiago, Chile. 200 p.

Lanino, M.; Holzapfel, E.

2012. Efecto de tres tasas de riego en el movimiento de sales en cultivo de melón (Cucumis melo) cultivar Galia, en microcanchones y en el camellón, en la Pampa del Tamarugal. Editorial Académica Española (EAE). España. 80 p.

Laucou, V.; Lacombe, T.; Dechesne, F.; Siret, R.; Bruno, J.P.; Dessup, M.; Dessup, T.; Ortigosa, P.; Parra, P.; Roux, C.; Santori, S.; Varés, D.; Peros, J.P.; Boursiquot, J.M.; This, T.

2011. High throughput analisis of grape genetic diversity as a tool for germoplasm collection management. Theor Appl Genet., 122: 1233-1245.

Novoa, R.; Villaseca, S.

1989. Mapa Agroclimático de Chile. Instituto de Investigaciones Agropecuarias. Ministerio de Agricultura. Chile. 216 p.

Núñez, L.

1985. Recuérdalo, aquí estaba el lagar: la expropiación de las aguas del valle de Quisma. Chungará, 14: 157-167.

Poblete, I.; Pinto, M.; Andrés, M.T.; M Hinrichsen, P.

2011. Genetic characterization of old grapevines collected in oases of the Atacama Desert. Chilean Journal of Agricultural Research, 71 (3): 476-482.

Poblete, I.; Vargas, D.; Lanino, M.; Zúñiga, A.

2017. Caracterización ampelográfica de la cepa Tamarugal (Vitis vinifera L.), originaria de la región de Tarapacá, desierto de Atacama. Idesia (35) 4: 47-54.

Ríaz, S.; De Lorenzis, G.; Velasco, D.; Koehmstedt, A.; Maghradze, D.; Bobokashvili, Z.; Musayev, M.; Zdnic, G.; Laucou, V.; Walker, M.; Failla, O.; Preece, J.; Aradhya, M.; Arroyo-García, R.

2018. Genetic diversity analisis of cultivated and wild grapevine (Vitis vinifera L.) accessions around the Mediterranean basin and central Asia. BMC Plant Biology, 18: $137-150$.

Rojas, G.

2015. Patrimonio e Identidad Vitivinícola: reflexiones sobre la evolución de los significados culturales del vino en Chile. RIVAR 2 (4): 88-105. 
Santibáñez, F.; Luzio, W.; Vera, W.; Etienne, M.; Lailhacar, S. 1982. Análisis de los ecosistemas de la I Región. Sacor Universidad de Chile. 195 p.

Thomas, M.R.; Scott, N.S.

1993. MicrosatelliteMicrosattelite repeats in grapevine reveal DNA polymorphims when analysed as sequence-tagged sites (STSs). Theor. Appl. Genet, 86: 985-990.
Toro-Lira, G.

2008. Las Viñas de Lima: los inicios de la vitivinicultura sudamericana 1539-1551. Editorial Universitaria Ricardo Palma. Lima, Perú. 90 p.

UPOV.

2008. Directrices para la ejecución del examen de la distinción, la estabilidad y la homogeneidad. Ginebra, Suiza. 53 p. UPOV.

2020. Miembros de la Unión Internacional para la protección de las Obtenciones Vegetales. Ginebra, Suiza. 2 p. 